\title{
Magnetic Monopoles and the Quantum Theory of Magnetism in Matter
}

\author{
Amagh Nduka \\ Federal University of Technology, Owerri, Nigeria \\ Email: amaghnduka@yahoo.com.au,ndukarp99@gmail.com
}

How to cite this paper: Nduka, A. (2018) Magnetic Monopoles and the Quantum Theory of Magnetism in Matter. Applied Mathematics, 9, 28-34.

https://doi.org/10.4236/am.2018.91003

Received: December 14, 2017

Accepted: January 20, 2018

Published: January 23, 2018

Copyright $\odot 2018$ by author and Scientific Research Publishing Inc. This work is licensed under the Creative Commons Attribution International License (CC BY 4.0).

http://creativecommons.org/licenses/by/4.0/

\section{c) (i) Open Access}

\begin{abstract}
Electricity and magnetism are common features of our world. The subject of electromagnetic fields in empty space populated only by point charges or smooth charge distributions in space is well understood. In that case, one deals with the classical theory of electrodynamics developed by J.C. Maxwell in 1864. Electromagnetism in the presence of matter is, however, a completely different problem. Microscopic electric behavior of homogeneous substances can in general be characterized fairly simply and completely. The theory that enables us to do this is fairly well understood. Sadly the situation is quite different for magnetism in matter. The study there is phenomenological. That is, the substance is characterized by a number of parameters and the experimentally determined relations among them. We are not aware of any successful microscopic theory of magnetism in matter. The microscopic theory of magnetic substances, a topic of fundamental and technological importance, is the subject of this paper.
\end{abstract}

\section{Keywords}

Monopoles, Diamagnetism, Paramagnetism, Ferromagnetism

\section{Introduction}

The history of magnetism dates back to the year 600 B.C. In that year, Thales of Miletus, often called the father of Greek science, detected magnetism in lodestone (magnetite). He discovered that lodestone attracts ordinary pieces of iron, and the iron itself becomes magnetized by touching the magnetite mineral. The Chinese discovered about the IIC that a magnet acts as a compass; and an important connection was made to the Chinese discovery by the discovery in $17 \mathrm{C}$ by William Gilbert that the earth is a magnet. These purely accidental observa- 
tions and many others of course, revealed the importance of magnetism to humanity.

We note here some interesting features of magnetism. The magnetic field outside a magnetized rod looks the same schematically like the electric field outside an electrically polarized rod. We infer from this observation that the magnetic field has a source which is related to it the way electric charge is related to electric field. This enabled Ampere to hypothesize that magnetism in matter is caused by a number of tiny rings of electric current distributed throughout the substance, implying that an ensemble of tiny rings of electric current is the source of magnetism.

As yet another bewildering observation let us put various substances into an intense magnetic field. It is found that for a large number of pure substances, force is observed. The force is found to be in the direction of the field for some substances and oppositely directed for others. This observation is independent of the direction of the magnetic field. Thus, some substances are attracted in the direction of increasing field intensity, and some others are repelled. Substances that are attracted in the direction of stronger field are called paramagnetic (e.g. $\mathrm{Al}, \mathrm{Na}$ ), and those that are repelled diamagnetic (e.g. $\mathrm{H}_{2} \mathrm{O}, \mathrm{NaCl}$ ). A third group consists of substances which behave like paramagnetic substances except that the force of attraction is overwhelmingly larger. These are called ferromagnetic substances. A fundamental question is this: What is there in the substance that makes it to be attracted or repelled on the application of a magnetic field?

We are all familiar with magnets, e.g. compass needle. In his autobiography Albert Einstein recalled the day his father bought a new toy for him. That toy was a compass needle, which aroused profound excitement in Einstein. Many youths shared in this excitement, and some experienced the fascination of horseshoe magnet which attracts iron. A striking feature of magnets is that if it is broken into two, each piece is also a magnet. Classically we can continue this process of subdivision of a magnet indefinitely producing ting magnets at each step right down to the atomic scale with electrons, etc. as magnets.

As our final example, but by no means exhaustive, we consider a striking feature of ferromagnetism which distinguishes it from diamagnetism and paramagnetism: A given ferromagnetic substance, e.g. pure iron, loses its ferromagnetism abruptly at a certain critical temperature called its Curie point. This transition temperature is different for different substances. It is $770^{\circ} \mathrm{C}$ for pure iron, $1150^{\circ} \mathrm{C}$ for pure cobalt, and $358^{\circ} \mathrm{C}$ for pure nickel. Conventional physics wisdom explains this in terms of magnetic domains, which is a purely non-microscopic concept.

The foregoing gives a few examples of common observable magnetic behavior. The study of such phenomena is generally, as has been noted, phenomenological. To date attempts to understand the atomic origin of various magnetic effects have not been satisfactory. In this paper, we give a complete and comprehensive quantum theory of magnetism in matter. 


\section{Magnetic Monopoles}

In an earlier paper we obtained the expression [1]

$$
\boldsymbol{\mu}_{q}=i\left(\frac{e m_{\ell}}{2 m_{e} c}\right) \hat{z}
$$

for the magnetic monopole (magnetic charge) of an electron in a substance. This expression shows that the magnetic monopole of an electron is clamped in direction determined by the cosmic direction " $\hat{z}$ ". Equation (1) shows that, like the electric charge, magnetic charge of the electron is quantized, with $\left(e / 2 \mathrm{~m}_{e} \mathrm{c}\right)$ as the magnetic charge quantum and $\mathrm{m}_{1}$ the magnetic quantum number. Thus, unlike the electric case where for a given charge $\mathrm{Q}=$ ne, the electric charge quantum number $\mathrm{n}$ takes all values, $\mathrm{n}=0, \pm 1, \pm 2, \cdots$, the quantum number $m_{\ell}$ takes only $(2 \ell+1)$ values for a given orbital quantum number $\ell$.

The classical treatment of this problem assumes that the orbiting electron is equivalent to a current ring with a far field that of a magnetic dipole having a dipole moment $\boldsymbol{m}$ given by [2],

$$
\boldsymbol{m}=\left(\frac{e}{2 m_{e} c}\right) \boldsymbol{L}
$$

The factor $\left(e / 2 \mathrm{~m}_{\mathrm{e}} \mathrm{c}\right)$, which we call the quantum of magnetic charge, is called there gyromagnetic ratio (or orbital magnetomechanical ratio) for the electron. It will become clear in the sequel why the term "magnetic moment" is inappropriate.

With $\vec{\mu}_{q}$ known, the microscopic magnetic field of an atomic electron in a substance is given by [1],

$$
\boldsymbol{B}=i\left(\frac{e m_{\ell}}{2 m_{e} c}\right) \hat{z} \times \boldsymbol{A}
$$

Up to this point the electron in the substance has been treated as a classical object of mass $m_{e}$ and negative charge $e$. In the quantum theory the electron must be treated as a quantum object having spin of magnitude $h / 2$ and "polarization" $(+=\hat{z}),(-=-\hat{z})$, or "unpolarized" with “+” or "-" polarizations equally probable in the absence of an external field. It follows from (1) that if the polarization is "+" and $m_{\ell}$ is positive the magnetic charge is negative and is given by

$$
\boldsymbol{\mu}_{q}=-i\left(\frac{e m_{\ell}}{2 m_{e} c}\right) \hat{z}
$$

and

$$
\boldsymbol{\mu}_{q}=i\left(\frac{e m_{\ell}}{2 m_{e} c}\right) \hat{z}
$$

if $m_{\ell}$ is negative. On the other hand if the polarization is "-" and $m_{\ell}$ is positive the magnetic charge is given by (5), and by (4) if $m_{\ell}$ is negative. Finally if 
the electron is unpolarized the atom becomes a magnetic dipole of charges $\pm \boldsymbol{\mu}_{q}$, with "separation distance" equal to $h$, where $h$ is Planck's constant. This follows from the fact that the unpolarized electron has "displacements" " $\pm(1 / 2 h) \hat{z}$ " so that the "separation distance" is $h$. This concept of a microscopic dipole, the tiniest magnet nature allows, makes it palpably clear why $\boldsymbol{\mu}_{q}$ is a charge and not a dipole moment vector.

Classically the simplest charge distribution with a dipole moment is two point charges, $+\mathrm{Q}$ and $-\mathrm{Q}$, separated by a distance $s$. The magnitude of the dipole moment vector is Qs and its direction points from the negative to the positive charge. Analogously our microscopic dipole moment vector has magnitude $h\left|\boldsymbol{\mu}_{q}\right|$, or $e h\left|m_{\ell}\right| / 2 m_{e} c=\left(0.9270 \times 10^{-20}\right)\left|m_{\ell}\right|$ ergs/gauss and direction “ $+\hat{z}$ ” or “ $-\hat{z}$ ". If $\left|m_{\ell}\right|=1$ the magnitude of the microscopic dipole moment is $0.9270 \times$ $10^{20}$ ergs/gauss, a quantity called Bohr magneton. It follows that what is called orbital g factor $\left(g_{1}\right)$ in elementary quantum theory is replaced by $\left|m_{\ell}\right|$ in the formal theory. As in the electric case, the force between the two charges of the magnetic dipole is attractive.

\section{Magnetic Substances}

There are three main types of magnetic behavior, namely, diamagnetism, paramagnetism, and ferromagnetism as has already been noted. What is called diamagnetism, paramagnetism, or ferromagnetism is only a matter of definition: If the two fundamental Equations (1) and (3) are simultaneously satisfied, the substance is ferromagnetic. In this case one detects magnetic effects in the neighborhood of the substance in the absence of an external magnetic field. On the other hand if only Equation (1) is satisfied and the substance experiences a force in the presence of an external magnetic field the substance is called diamagnetic or paramagnetic. It is diamagnetic if the substance is repelled by external magnetic field, and paramagnetic if the force is attractive. It follows that diamagnetism or paramagnetism is an intrinsic property. Those substances that do not satisfy Equation (1) are called non-magnetic substances. We note from Equations (1) and (3) that all substances for which $m_{\ell}=0$ are non-magnetic, it follows from these two equations that $m_{\ell} \neq 0$ for magnetic substances.

\subsection{Diamagnetism and Paramagnetism}

Consider an ensemble consisting of a large number $(\mathrm{N})$ of one electron magnetic atoms each of which is polarized either in the " $+\hat{z}$ " or " $-\hat{z}$ " direction in equilibrium at a temperature $\mathrm{T}^{0} \mathrm{~K}$. If the electron has polarization " $+\hat{z}$ " and $m_{\ell}$ is positive, $\boldsymbol{\mu}_{q}$ is given by (4), $\boldsymbol{\mu}_{q}$ is given by (5) if $m_{\ell}$ is negative. Similar considerations apply if the polarization is " $-\hat{z}$ ". The force $\left(\mathrm{F}_{z}\right)$ on the monopole in the presence of an external field $\boldsymbol{B}$ is given by [2].

$$
F_{z}=\mu_{q} \cdot \nabla B_{z},
$$

which is positive if $\boldsymbol{\mu}_{q}$ is given by (5) and negative if $\boldsymbol{\mu}_{q}$ is given by (4), pro- 
vided only Equation (1) is satisfied. The total force on the substance is given by $\mathrm{NF}_{z}$. Thus, a substance is called paramagnetic if it contains monopoles oriented in the direction of " $\hat{z}$ " and diamagnetic if it contains monopoles oriented in the direction " $-\hat{z}$ ".

\subsection{Ferromagnetism}

If in the foregoing analysis of section 3.1 the substance exhibits magnetic effects in the absence of any external magnetic field it is called ferromagnetic. Since all the monopoles of the $\mathrm{N}$ atoms are oriented either in the " $+\hat{z}$ " or " $-\hat{z}$ " direction the substance is called a permanent magnet.

Let us now consider an ensemble consisting of a large number $(\mathrm{N})$ of unpolarized magnetic atoms in equilibrium at a temperature $T^{0} \mathrm{~K}$ which is below the Curie point of the substance. In this case the two polarizations " $+\hat{z}$ " and " $-\hat{z}$ " are equally probable, and so each atom becomes, as already explained, a microscopic dipole so that the system becomes an ensemble of a large number $(N)$ of magnetic dipoles with random orientation such that $50 \%$ of them point in the " $+\hat{z}$ " direction and $50 \%$ in the " $-\hat{z}$ " direction. We say that the substance is unmagnetized. In the presence of a strong external magnetic field the substance becomes magnetized with $100 \%$ of the dipoles in the " $+\hat{z}$ " or " $-\hat{z}$ " direction, e.g. magnetized piece of iron.

The magnetization process described here is an unusual one. Classically one expects dipoles oriented in a direction opposite to that of the external magnetic field to undergo infinitesimal rotations in angular momentum space about an axis perpendicular to the plane containing the magnetic field as the field strength increases to bring it in alignment with the direction of the field. This is not so in the quantum case because of the non-commutativity of the angular momentum components. The allowed process in this case is what is called "quantum flips", i.e. quantum jumps from, for example, " $-\hat{z}$ " to " $+\hat{z}$ " direction and conversely. Thus, as $\boldsymbol{B}$ increases from $\boldsymbol{B}=0$, the dipoles oriented in a direction opposite to that of the field undergo continuous quantum flips until all the dipoles are oriented parallel to the field, the substance acquiring magnetization $\boldsymbol{M}$. The physical reason for these quantum flips is to ensure that the energy of the magnetized substance is minimized.

As we earlier noted a given ferromagnetic substance loses its ferromagnetic properties abruptly if heated to a certain critical temperature (Tc) called its $\mathrm{Cu}$ rie point. The Curie point is different for different substances. It is $770^{\circ} \mathrm{C}$, $1150^{\circ} \mathrm{C}$, and $358^{\circ} \mathrm{C}$ for pure iron, cobalt, and nickel respectively. The different Curie points for substances have their origin in the quantum theory, because it depends on the quantum number $m_{\ell}, m_{\ell}$ is $2,-2,-1$ for iron, cobalt, and nickel respectively [3].

At temperatures above the Curie point the ensemble of magnetic dipoles becomes an ensemble of unpolarized magnetic atoms in equilibrium at the given temperature. In the presence of an external magnetic field each atom of the en- 
semble exhibits both diamagnetic and paramagnetic behavior, but because the paramagnetic effect is larger we say that the substance becomes paramagnetic. If the substance is again cooled below its Curie point it becomes ferromagnetic because each atom becomes a dipole.

Formally the Curie effect is due to the absorption of infrared radiation by the atoms of the substance at a frequency $v_{c}$ given by the Planck condition

$$
v_{c}=\frac{k T c}{h},
$$

which heats up the substance. As a result the bond (i.e. the attractive magnetostatic force) between the magnetic charges of the dipoles is broken. $v_{c}$ is about $2.2124 \times 10^{12} \mathrm{cps}, 3.0185 \times 10^{12} \mathrm{cps}$, and $1.3385 \times 10^{12} \mathrm{cps}$ for iron, cobalt, and nickel respectively.

\section{Conclusions}

Our quantum theory of magnetism in matter presented here has opened up new vistas in our understanding of magnetism in matter. We limit our discussion here to a small sample of its consequences.

Our first task is to explain why one does not observe magnetic effects in the neighbourhood of an infinite rod of unmagnetized ferromagnetic substance, e.g. iron, and why the magnetic field outside an unmagnetized rod of finite length looks the same as that of the electric field outside an electric dipole consisting of two point charges, $+Q$ and $-Q$. An infinite rod of unmagnetized substance is an ensemble of randomly oriented magnetic dipoles having $50 \%$ of its dipoles pointing in the " $+\hat{z}$ " direction and $50 \%$ in the " $-\hat{z}$ " direction. Such a sample is magnetically neutral. For unmagnetized rods of finite extent magnetic charges accumulate at the ends as positive magnetic charge (or "north pole") at one end and negative magnetic charge (or "south pole") at the other end. The accumulation of magnetic charges at the ends is an edge effect caused by the fact that only magnetic dipoles of a particular orientation survive at the ends. Away from the ends, the magnetic dipoles cancel out exactly because of opposite orientation and the rod is magnetically neutral there. Such a finite rod is called a magnet. The magnetic field outside such an object is the field of a magnet which is known to be the same as that of an electrically polarized rod, or that of two point electrical charges of opposite sign separated by a small distance $\mathrm{s}$.

If the finite rod is cut into two pieces, we obtain two magnets for the same reason as above. This process of subdivision is not indefinite, and it continues down to the microscopic magnet having positive magnetic charge at one end and negative magnetic charge at the other end separated by "distance" $h$, where $\mathrm{h}$ is Planck's constant.

Geomagnetism is the science concerned with earth's magnetic field. The field extends far above the surface of the earth. The source of the field is, however, a mystery; but geophysicists believe that it is associated with dynamo action in the earth's liquid core! [4] The content of the above paragraph resolves the problem 
completely and unambiguously. The earth is a finite body (oblate spheroid) containing a large number of randomly oriented magnetic dipoles. In other words, the earth is a magnet and hence its magnetic field. The orientation of the magnet is such that its "north pole" is located near the earth's geographic south pole and its "south pole" is located near the earth's geographic north pole. The reason for this orientation is not difficult to understand. Near the geographic south pole, the magnetic dipoles pointing in the southerly direction (" $-\hat{z}$ ") are missing, hence the accumulation of positive magnetic charges there, and near the geographic north pole, the magnetic dipoles pointing in the northerly direction are missing, hence the accumulation of negative magnetic charges there.

We have seen from this work that electrons endowed with magnetic charge reside in "angular momentum space". This makes it clear that these electrons are fundamentally distinct from electrons endowed with electric charge because, as can be seen from the equations of quantum electrodynamics [1], electric charge electrons reside in momentum space. We deduce that magnetism is the only known physical phenomenon that violates Einstein's principle of relativity in that magnetic charge electrons reside in non-inertial frame of reference. It follows that Einstein's principle of relativity cannot be regarded as sacrosanct in the formulation of the theories of the fundamental processes.

\section{References}

[1] Nduka, A. (2017) Magnetic Monopoles. Applied Mathematics, 8, 245-251. https://doi.org/10.4236/am.2017.82020

[2] Purcell, E.M. (1963) Berkeley Physics Course. Volume 2, McGraw-Hill, New York.

[3] Nduka, A. (2014) The Geometrical Theory of the Structure of Nuclei, Atoms, and Molecules. Applied Mathematics, 5, 2209-2215. https://doi.org/10.4236/am.2014.515214

[4] Physics Today (2016) Physics Update, p. 25. 